\title{
Propriétés rhéologiques de farines panifiables formulées à partir d'extraits d'amidon et de gluten de blé
}

\author{
Paulin AZOKPOTA ${ }^{1 *}$, Marianne SINDIC ${ }^{2}$, Michel PAQUOT ${ }^{2}$ et Claude DEROANNE $^{2}$ \\ ${ }^{1}$ Université d'Abomey - Calavi, Faculté des Sciences Agronomiques, Département de Nutrition, Sciences et \\ Technologies Alimentaires, 01. BP. 526. Cotonou. République du Bénin. \\ ${ }^{2}$ Faculté Universitaire des Sciences Agronomiques de Gembloux, Unité de Technologie des Industries \\ Alimentaires. 34, Rue des Déportés. Gembloux, Belgique. \\ *Auteur correspondant ; E-mail : azokpotap@yahoo.fr ; paulin.azokpota@fsa.uac.bj
}

\section{RESUME}

Le présent travail a mis en évidence les propriétés rhéologiques des farines panifiables reconstituées avec des extraits d'amidon. Du gluten vital $10 \%$ a été incorporé à $90 \%$ d'extraits d'amidons de blé, de maïs, de riz et de la pomme de terre pour obtenir, respectivement, quatre (4) types de farines F1, F2, F3 et F4. Les farines ont été reconstituées à l'aide d'un mélangeur de type KENWOOD à 4 unités de vitesse. Les caractéristiques rhéologiques des pâtes des farines reconstituées ont été analysées à l'aide du Farinographe Brabender et de l'Alvéographe Chopin. Les farines F2, F3 et F4 sont caractérisées par une forte capacité d'hydratation, une faible activité $\alpha$-amylasique qui se traduit par des temps de chute très élevés. Il en résulte des pâtes de très faible tenue avec une résistance à l'extraction, en comparaison avec une farine de référence (Fo). La force de la pâte de la farine $\mathrm{F} 1$ est plus élevée $\left(\mathrm{W}=85.10^{-4} \mathrm{~J}\right)$ que celle des pâtes des farines F2, F3 et F4. Globalement, l'équilibre de configuration $(\mathrm{P} / \mathrm{L})$ entre la ténacité et l'élasticité des pâtes des farines est de l'ordre de 0,88 alors que les indices d'élasticité varient entre 7,3 et 9,4.

(c) 2010 International Formulae Group. All rights reserved.

Mots clés : Amidons ; Farines panifiées ; Gluten vital ; Caractéristiques rhéologiques

\section{INTRODUCTION}

La consommation des produits de boulangerie, notamment celle du pain occupe une place prépondérante dans le régime alimentaire des peuples, même dans les pays non producteurs de blé. Ces derniers deviennent de plus en plus tributaires des nations productrices de blé, particulièrement lors des crises économiques où le blé revient très cher à l'importation. Pour palier cette situation, il se développe de plus en plus des technologies de substitution de la farine de blé par des farines provenant des ressources alimentaires locales (Defloor, 1995; Balla, 1999). En effet, les farines panifiables reconstituées sont généralement des mélanges de farines de blé incorporées de farines de céréales (riz, maïs, sorgho, etc.) ou de racines et tubercules (pomme de terre, manioc, etc.) dans des proportions variées (Asiedu, 1991; CTA, 1991 ; Melcion, 1991 ; Balla, 1999). La farine de blé est composée environ, de $90 \%$ d'amidon et de $10 \%$ de gluten (Dobraszczyk and Morgenstern, 2003). Du point de vue pondéral, l'amidon constitue donc la partie prépondérante de la farine de blé et la qualité 
des pâtes des farines reconstituées dépend de celle du gluten du blé certes, mais essentiellement de la qualité des amidons des farines récurrentes (Melcion, 1991; Lefebvre et al., 2000 ; Uthayakumaran et al., 2002). En effet, l'impact des amidons gélatinisés sur les propriétés rhéologiques de la pâte des farines a été rapporté par de nombreux auteurs (Mestres, 1986 ; Chaunier et al., 2005) ainsi que leur influence sur la qualité des produits panifiés obtenus (Buléon et al., 1990 ; Berton et al., 2002).

Cependant, à notre connaissance, les travaux de recherche sur la formulation et la caractérisation des farines panifiables à partir des ressources alimentaires locales, autres que le blé ne prennent toujours pas en compte l'étude des propriétés rhéologiques des amidons des farines récurrentes, pris séparément. La possibilité de formuler des farines panifiables à partir des amidons de différentes sources alimentaires incorporés de gluten de blé constitue une préoccupation. La question de recherche pour le présent travail est de s'interroger sur la possibilité d'obtenir des farines panifiables en incorporant du gluten de blé à l'amidon provenant de n'importe quelle source alimentaire.

Le présent travail a pour objectif principal de mettre en évidence l'interaction gluten-amidon dans la formulation de farines panifiables. Les objectifs spécifiques de l'étude visent à :

- déterminer les caractéristiques rhéologiques de différents amidons d'usage courant;

- caractériser, aux plans physico-chimiques et rhéologiques les farines reconstituées à partir des amidons incorporés de gluten.

\section{MATERIEL ET METHODES Matériel}

Les farines ont été reconstituées à partir des amidons de blé, de maïs, de pomme de terre et de riz, et du gluten vital. Tous les amidons ont été fournis par les sociétés COSUCRA (Fontenoy, Belgique) et REMY INDUSTRIES (Wijgmaal-Leuven, Belgique) tandis que le gluten vital a été fourni par la société AMYLUM GROUP (Aalst, Belgique). La farine de blé (12,5/680, RC. Huy 81, de moulins de State-Huy, Belgique) a été utilisée comme farine de référence (Fo).

\section{Méthodes}

Caractérisation physico-chimique et rhéologique des amidons

Les matières sèches et les cendres des amidons ont été déterminées suivant la méthode NF V03-707 (AFNOR, 1991). Les protéines brutes ont été dosées par la méthode Kjeldahl (AACC, 1983 ; Method 44-15). La détermination de la teneur en matières grasses a été effectuée selon la méthode d'extraction à l'éther par pesée différentielle (AACC, 1983 ; Method 30-25). La température initiale $\left(\mathrm{T}_{0}\right)$, la température de gonflement $\left(\mathrm{T}_{\mathrm{e}}\right), \quad$ la température de gélification $\left(\mathrm{T}_{\mathrm{g}}\right)$, la température finale $\left(\mathrm{T}_{\text {fin }}\right)$, la viscosité initiale $\left(\mathrm{V}_{0}\right)$, la viscosité maximale $\left(\mathrm{V}_{\max }\right)$ au cours du chauffage, la viscosité après 20 minutes de chauffage à $97^{\circ} \mathrm{C}\left(\mathrm{V}_{97 \mathrm{f}}\right)$, la Viscosité après la phase de refroidissement $\left(\mathrm{V}_{\text {fin }}\right)$, la Viscosité de gonflement $\left(\mathrm{V}_{\mathrm{e}}\right)$, la viscosité de gélification $\left(\mathrm{V}_{\mathrm{g}}\right)$, l'indice de gélatinisation $\left(\mathrm{V}_{\mathrm{fin}}-\mathrm{V}_{97 \mathrm{f}}\right)$ et l'indice de stabilité de l'amidon $\left(\mathrm{V}_{\max }-\mathrm{V}_{97 \mathrm{f}}\right)$ ont été déterminés selon la méthode de Nago (1997). L'activité $\alpha$-amylasique a été déterminée par la méthode NF V03-703 (AFNOR, 1991).

\section{Reconstitution des farines}

Le gluten, dans une proportion de $10 \%$ (P/P, base sèche), a été incorporé à $90 \%$ (base sèche) de chaque amidon. Ainsi, pour $100 \mathrm{~g}$ de farine constituée, $90 \mathrm{~g}$ de chaque type d'amidon et $10 \mathrm{~g}$ de gluten vital, en base sèche, ont été mélangés. Le mélange a été homogénéisé à l'aide d'un mixeur de type 
KENWOOD à 4 unités de vitesse. Quatre types de farines ont été ainsi reconstitués, à savoir, les farines reconstituées à base des amidons de blé (F1), de maïs (F2), de riz (F3) et de la pomme de terre (F4). Pour la maturation, les farines ainsi reconstituées ont été laissées au repos, pendant une semaine, en chambre froide à $15{ }^{\circ} \mathrm{C}$, avant d'être analysées.

\section{Caractérisation physico-chimique et} rhéologique des farines reconstituées

Les caractéristiques physico-chimiques des farines ont été déterminées en utilisant les mêmes méthodes que pour les amidons. Les paramètres de viscosité des farines reconstituées ont été mesurés à l'aide du viscoamylographe Brabender (OHG Duisburg-Germany, type 801202). Le principe de la méthode repose sur l'enregistrement de la résistance d'une suspension de farine sous agitation constante avec élévation de température. Les mesures des paramètres ont été effectuées sur des suspensions de farine à $8 \%$ selon les méthodes utilisées par Hounhouigan (1994) et Nago (1997). Par ailleurs, la capacité d'hydratation des farines a été évaluée à l'aide d'un Farinographe de Brabender (AACC, 1983 ; Method 54-29) de même que la tolérance au pétrissage (AACC ; 1984 ; Method 54-21). Le comportement à la déformation des pâtes des farines reconstituées a été évalué à l'aide de l'alvéographe de Chopin (AACC, 1983 ; Method 54-30). La solubilité est déterminée sur des suspensions aqueuses à $8 \%$ de matière sèche selon la méthode de Nago (1997).

\section{Analyses statistiques}

Les données relatives à la composition chimique et aux caractéristiques rhéologiques des amidons et des farines reconstituées ont été soumises à l'analyse de variance (ANOVA) avec le logiciel Statistica 7
(StatSoft, Tulsa, USA), modèle fixe à chacun des deux facteurs pris séparément, à savoir la composition chimique, d'une part, et les propriétés rhéologiques, d'autre part. Les conditions préalables à l'ANOVA (normalité et égalité de variances) ont été vérifiées. Le test de Student-Newman et Keuls a été effectué sur chaque variable pour structurer les moyennes des différentes modalités par rapport à la composition chimique et aux propriétés rhéologiques.

\section{RESULTATS}

\section{Caractéristiques physico-chimiques et rhéologiques des amidons \\ Le Tableau 1 présente les} caractéristiques physico-chimiques des quatre types d'amidon analysés. Au regard des résultats obtenus, il n'y a pas de différence significative $(P>0,05)$ entre les valeurs des indices de solubilité des amidons de blé et de riz. L'amidon de maïs est caractérisé par l'indice de solubilité le plus élevé, celui de la pomme de terre étant le plus faible. Les mêmes observations ont été faites concernant les valeurs de la matière solubilisée pour les quatre types d'amidon.

Les quatre types d'amidon analysés présentent différentes valeurs de temps de chute avec une différence significative $(\mathrm{P}<$ 0,05). L'amidon de la pomme de terre présente un temps de chute plus élevé (442 s), ce qui correspondrait à une faible activité $\alpha$ amylasique, tandis que l'amidon de blé a un temps de chute plus court (338 s), ce qui se traduirait par une plus forte activité $\alpha$ amylasique.

Sur la base du profil des viscogrammes caractérisant les principales valeurs de viscosité, en fonction du temps de traitement thermique, les principales propriétés rhéologiques des amidons ont été déterminées, à savoir: les indices de gélatinisation et de 
stabilité des amidons ; les viscosités maximale $\left(\mathrm{V}_{\max }\right.$ et finale $\left(\mathrm{V}_{\mathrm{fin}}\right)$; les viscosités au début de $97{ }^{\circ} \mathrm{C}\left(\mathrm{V}_{97}\right)$ et à la fin de $97{ }^{\circ} \mathrm{C}\left(\mathrm{V}_{97 \mathrm{f}}\right)$; les températures de gélatinisation et de gonflement et les viscosités correspondantes (Tableau 2). L'amidon de la pomme de terre a une viscosité maximale plus élevée (1840 \pm $25 \mathrm{UB})$, un indice de gélatinisation plus élevé $(1070 \pm 30 \mathrm{UB})$ et un indice de stabilité de l'ordre de $960 \pm 12$ UB. En outre, les amidons de blé, de maïs et de riz sont caractérisés par des indices de stabilité, respectivement, de l'ordre de 100, 505 et 107 UB (Tableau 2). La viscosité maximale de l'amidon de blé est de 310 UB.

\section{Caractéristiques physico-chimiques et rhéologiques des farines reconstituées à partir des amidons analysés}

Globalement, les caractéristiques physico-chimiques des farines reconstituées à partir des amidons de céréales (F1, F2, F3) sont similaires mais diffèrent de celles de la farine reconstituée à partir de l'amidon de la pomme de terre (F4) (Tableau 3). Les farines reconstituées à base des amidons présentent une faible activité $\alpha$-amylasique qui se traduit par des temps de chute très élevés par rapport à la farine de référence (Fo). Seule, la farine F1 a un temps de chute sensiblement proche de celle de la farine référence.

Les principaux paramètres déterminant les caractéristiques des farines reconstituées sont mentionnés dans le Tableau 4. Seules, les caractéristiques rhéologiques des pâtes des farines F0 et F1 ont pu être mesurées. Les valeurs de la force des farines (W) caractérisant le travail de la déformation de la pâte jusqu'à la rupture est de l'ordre de $109,5 \cdot 10^{-4} \mathrm{~J}$ pour la farine F0 et de $85,10.10^{-4}$ $\mathrm{J}$ pour la farine F1 (Tableau 4). Le rapport de configuration $(\mathrm{P} / \mathrm{L})$ traduisant l'équilibre entre la ténacité et l'élasticité de la pâte est 0,35 pour la farine $\mathrm{F} 0$ et 0,88 pour la farine $\mathrm{F} 1$.

Tableau 1 : Caractéristiques physico-chimiques des amidons*.

\begin{tabular}{lccccccc}
\hline $\begin{array}{c}\text { Echantillons } \\
\text { d'amidon }\end{array}$ & MS & PB & IS $^{1}$ & MS $^{2}$ & MG & CT & THg \\
\hline AB & $88 \pm 1,3 \mathrm{a}$ & $0,24 \pm 0,01 \mathrm{a}$ & $7,8 \pm 0,5 \mathrm{~b}$ & $0,007 \pm 0,001 \mathrm{~b}$ & $0,01 \pm 0,001 \mathrm{c}$ & $0,07 \pm 0,001 \mathrm{~b}$ & $338 \pm 2,4 \mathrm{~d}$ \\
$\mathrm{AM}$ & $89 \pm 1,2 \mathrm{a}$ & $0,21 \pm 0,03 \mathrm{a}$ & $9,4 \pm 0,7 \mathrm{a}$ & $0,009 \pm 0,002 \mathrm{a}$ & $0,23 \pm 0,003 \mathrm{a}$ & $0,07 \pm 0,005 \mathrm{~b}$ & $362 \pm 2,7 \mathrm{~b}$ \\
$\mathrm{AP}$ & $82 \pm 0,9 \mathrm{~b}$ & $0,05 \pm 0,002 \mathrm{~b}$ & $7,3 \pm 0,7 \mathrm{c}$ & $0,0006 \pm 0,001 \mathrm{~b}$ & $0,10 \pm 0,05 \mathrm{~b}$ & $0,05 \pm 0,001 \mathrm{c}$ & $442 \pm 4,2 \mathrm{a}$ \\
$\mathrm{AR}$ & $88 \pm 0,8 \mathrm{a}$ & $0,23 \pm 0,02 \mathrm{a}$ & $8 \pm 0,3 \mathrm{~b}$ & $0,007 \pm 0,002 \mathrm{~b}$ & $0,14 \pm 0,02 \mathrm{~b}$ & $0,11 \pm 0,05 \mathrm{a}$ & $360 \pm 3,3 \mathrm{c}$ \\
\hline
\end{tabular}

\footnotetext{
": Moyennes \pm Ecarts Types des résultats de trois répétitions effectuées à trois différentes occasions. Les moyennes précédées de la même lettre dans la même colonne ne sont pas significativement différentes $(\mathrm{P}>0,05)$, pour un paramètre donné.

${ }^{1}$ : Indice de solubilité $;^{2}$ : Teneur en matière solubilisée ; AB : Amidon de blé ; AM : Amidon de maïs ; AP : Amidon de pomme de terre ; AR : Amidon de riz; MS : Matières sèches (\%, base humide); PB : Protéines brutes (\%, base sèche) ; MG : Matières grasses (\%, base sèche); THg : Temps de chute Hagberg (s) ; CT : Cendres Totales ;
} 
Tableau 2 : Caractéristiques rhéologiques des amidons*.

\begin{tabular}{lcccc}
\hline Caractéristiques rhéologiques & \multicolumn{4}{c}{ Echantillons d'amidon } \\
\cline { 2 - 5 } & $\mathbf{A B}$ & $\mathbf{A M}$ & $\mathbf{A R}$ & $\mathbf{A P}$ \\
\hline $\mathrm{V}_{\max }(\mathrm{UB})$ & $310 \pm 15 \mathrm{~d}$ & $510 \pm 34 \mathrm{~b}$ & $394 \pm 17 \mathrm{c}$ & $1840 \pm 25 \mathrm{a}$ \\
$\mathrm{V}_{97} \mathrm{f}(\mathrm{UB})$ & $210 \pm 11 \mathrm{~d}$ & $405 \pm 10 \mathrm{~b}$ & $287 \pm 10 \mathrm{c}$ & $880 \pm 18 \mathrm{a}$ \\
$\mathrm{V}_{\text {fin }}(\mathrm{UB})$ & $950 \pm 22 \mathrm{c}$ & $1210 \pm 30 \mathrm{~b}$ & $564 \pm 14 \mathrm{~d}$ & $1950 \pm 27 \mathrm{a}$ \\
$\mathrm{V}_{\max -\mathrm{Vfin}}(\mathrm{UB})$ & $100 \pm 7 \mathrm{~b}$ & $105 \pm 10 \mathrm{~b}$ & $107 \pm 11 \mathrm{~b}$ & $960 \pm 12 \mathrm{a}$ \\
$\mathrm{V}_{\text {fin-Vmax }}(\mathrm{UB})$ & $740 \pm 12 \mathrm{~b}$ & $700 \pm 19 \mathrm{~b}$ & $277 \pm 14$ & $1070 \pm 30 \mathrm{a}$ \\
$\mathrm{V}_{\mathrm{T} 0}(\mathrm{UB})$ & $20 \pm 2 \mathrm{~b}$ & $30 \pm 4 \mathrm{a}$ & $30 \pm 7 \mathrm{a}$ & $30 \pm 4 \mathrm{a}$ \\
$\mathrm{V}_{\mathrm{Te}}(\mathrm{UB})$ & $34 \pm 1,5 \mathrm{~b}$ & $50 \pm 2 \mathrm{a}$ & $50 \pm 2 \mathrm{a}$ & $50 \pm 3 \mathrm{a}$ \\
$\mathrm{V}_{\mathrm{Tg}}(\mathrm{UB})$ & $192 \pm 10 \mathrm{~d}$ & $520 \pm 14 \mathrm{~b}$ & $400 \pm 11 \mathrm{c}$ & $1000 \pm 22 \mathrm{a}$ \\
$\mathrm{T}_{0}\left({ }^{\circ} \mathrm{C}\right)($ fixe$)$ & 25 & 25 & 25 & 25 \\
$\mathrm{~T}_{\mathrm{e}}\left({ }^{\circ} \mathrm{C}\right)$ & $71,5 \pm 5 \mathrm{a}$ & $70,5 \pm 11 \mathrm{a}$ & $70,5 \pm 10 \mathrm{a}$ & $61 \pm 7 \mathrm{~b}$ \\
$\mathrm{~T}_{\mathrm{g}}\left({ }^{\circ} \mathrm{C}\right)$ & $80 \pm 4 \mathrm{a}$ & $78,5 \pm 3 \mathrm{a}$ & $78,75 \pm 8 \mathrm{a}$ & $65 \pm 7 \mathrm{~b}$ \\
$\mathrm{~T}_{\text {fin }}\left({ }^{\circ} \mathrm{C}\right)$ (fixe) & 20 & 20 & 20 & 20
\end{tabular}

$\mathrm{T}_{0}$ : Température initiale $; \mathrm{T}_{\mathrm{e}}:$ Température de gonflement $; \mathrm{T}_{\mathrm{g}}:$ Température de gélification $; \mathrm{T}_{\mathrm{fin}}:$ Température finale $; \mathrm{V}_{\max }:$ Viscosité maximale au cours du chauffage $; \mathrm{V}_{97 \mathrm{f}}:$ Viscosité après $20 \mathrm{minutes}$ de

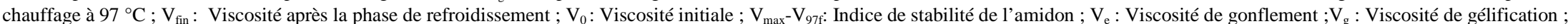

$\mathrm{V}_{\text {fin }}-\mathrm{V}_{97 \mathrm{f}}$ : Indice de gélatinisation; $\mathrm{AB}, \mathrm{AM}, \mathrm{AR}$, AP: respectivement, amidons de blé, de maïs, de riz et de pomme de terre ; UB : Unité de Brabender;

*: Moyennes \pm Ecarts Types des résultats de trois répétitions effectuées à trois différentes occasions. Les moyennes précédées de la même lettre sur la même ligne ne sont pas significativement différentes $(\mathrm{P}>0,05)$ pour un paramètre donné. 
P. AZOKPOTA et al. / Int. J. Biol. Chem. Sci. 4(5): 1582-1592, 2010

Tableau 3 : Caractéristiques physico-chimiques des farines reconstituées*.

\begin{tabular}{|c|c|c|c|c|c|c|c|c|}
\hline \multirow[t]{2}{*}{ FR } & \multicolumn{4}{|c|}{ Composition chimique } & \multicolumn{3}{|c|}{ Couleur } & \multirow[t]{2}{*}{ THg (S) } \\
\hline & MS & PB & MG & CT & $\mathbf{L}^{*}$ & $\mathbf{a}^{*}$ & $\mathbf{b}^{*}$ & \\
\hline F0 & $85,5 \pm 0,9 \mathrm{c}$ & $9,82 \pm 0,8 \mathrm{~b}$ & $1,47 \pm 0,02 \mathrm{a}$ & $1,52 \pm 0,02 \mathrm{a}$ & $87,66 \pm 1,2 \mathrm{~b}$ & $1,07 \pm 0,05 \mathrm{a}$ & $15,12 \pm 0,3 \mathrm{a}$ & $60 \pm 2,3 \mathrm{e}$ \\
\hline $\mathrm{F} 1$ & $88 \pm 1,2 \mathrm{~b}$ & $9,75 \pm 0,7 \mathrm{~b}$ & $1,52 \pm 0,02 \mathrm{a}$ & $1,37 \pm 0,04 \mathrm{a}$ & $93,32 \pm 1,4$ a & $1,02 \pm 0,02 \mathrm{a}$ & $14,62 \pm 0,5 \mathrm{ab}$ & $102 \pm 3,5 \mathrm{~d}$ \\
\hline $\mathrm{F} 2$ & $89 \pm 1,3 \mathrm{~b}$ & $9,04 \pm 0,3 \mathrm{~b}$ & $1,72 \pm 0,03 \mathrm{a}$ & $1,36 \pm 0,02 \mathrm{a}$ & $94,44 \pm 1,5 \mathrm{a}$ & $1,12 \pm 0,01 \mathrm{a}$ & $13,06 \pm 0,2 \mathrm{c}$ & $114 \pm 1,5 \mathrm{a}$ \\
\hline F3 & $88 \pm 1,1 \mathrm{~b}$ & $9,50 \pm 0,4 \mathrm{~b}$ & $1,60 \pm 0,02 \mathrm{a}$ & $1,33 \pm 0,01 \mathrm{a}$ & $94,58 \pm 1,7 \mathrm{a}$ & $1,10 \pm 0,02 \mathrm{a}$ & $13,04 \pm 0,6 \mathrm{c}$ & $128 \pm 1,8 \mathrm{c}$ \\
\hline F4 & $83 \pm 1,2 \mathrm{c}$ & $8,25 \pm 0,3 \mathrm{c}$ & $1,64 \pm 0,01 \mathrm{a}$ & $1,40 \pm 0,02 \mathrm{a}$ & $94,58 \pm 1,3 \mathrm{a}$ & $1,03 \pm 0,03 \mathrm{a}$ & $13,05 \pm 0,8 \mathrm{c}$ & $142 \pm 3,2 \mathrm{~b}$ \\
\hline GV & $92,6 \pm 0,4 \mathrm{a}$ & $83,21 \pm 0,5 \mathrm{a}$ & $1,35 \pm 0,02 \mathrm{a}$ & $0,92 \pm 0,02 \mathrm{~b}$ & $87,58 \pm 0,8 \mathrm{~b}$ & $1,13 \pm 0,04 \mathrm{a}$ & $16,02 \pm 0,7 \mathrm{a}$ & $62 \pm 1,2 \mathrm{e}$ \\
\hline
\end{tabular}

${ }^{*}$ : Moyennes \pm Ecarts Types des résultats de trois répétitions effectuées à trois différentes occasions. Les moyennes précédées de la même lettre dans la même colonne ne sont pas significativement différentes $(\mathrm{P}>0,05)$, pour un paramètre analysé : F0: Farine de blé de référence; F1: Farine reconstituée avec l'amidon de blé et le gluten vital; F2: Farine reconstituée avec l'amidon de maïs et le gluten vital; F3: Farine reconstituée avec l'amidon de riz et le gluten vital; F4 Farine reconstituée avec l'amidon de pomme de terre et le gluten vital ; L*: Luminance indiquant la clarté;

a*: Indice de saturation en rouge ; $b^{*}$ : Indice de saturation en jaune; FR: Farines Reconstituées ; GV: Gluten vital; MS, PB, MG, CT, THg (Cf. Tableau 1).

Tableau 4 : Caractéristiques rhéologiques des farines reconstituées*.

\begin{tabular}{lcccccc}
\hline & \multicolumn{6}{c}{ Paramètres rhéologiques analysés } \\
\cline { 2 - 7 } FR & $\mathbf{P}(\mathbf{m m})$ & $\mathbf{L}(\mathbf{m m})$ & $\mathbf{P} / \mathbf{L}$ & $\mathbf{W}\left(\mathbf{1 0}^{-4} \mathbf{J}\right)$ & $\mathbf{I S}^{\mathbf{1}}(\boldsymbol{\%})$ & $\mathbf{M S}^{2}(\mathbf{g} / \mathbf{m l})$ \\
\hline Fo & $28,9 \pm 8 \mathrm{~b}$ & $82,56 \pm 19 \mathrm{a}$ & $0,35 \pm 0,03 \mathrm{~b}$ & $109,5 \pm 25 \mathrm{a}$ & $7,8 \pm 0,5 \mathrm{~b}$ & $0,007 \pm 0,001 \mathrm{a}$ \\
F1 & $67,02 \pm 15 \mathrm{a}$ & $76,22 \pm 12 \mathrm{~b}$ & $0,88 \pm 0,01 \mathrm{a}$ & $85,10 \pm 13 \mathrm{~b}$ & $9,4 \pm 0,7 \mathrm{a}$ & $0,009 \pm 0,002 \mathrm{a}$ \\
F2 & - & - & - & - & $7,1 \pm 0,7 \mathrm{c}$ & $0,0006 \pm 0,001 \mathrm{~b}$ \\
F3 & - & - & - & - & $8,0 \pm 0,3 \mathrm{~b}$ & $0,007 \pm 0,002 \mathrm{a}$ \\
F4 & - & - & - & - & - & -
\end{tabular}

${ }^{*}:$ Moyennes \pm Ecarts Types des résultats de trois répétitions effectuées à trois différentes occasions. Les moyennes précédées de la même lettre dans la même colonne ne sont pas significativement différentes $(\mathrm{P}>0,05)$, pour un paramètre analysé ; $\mathrm{P}$ : Ténacité de la pâte, pression au point de rupture (en mm d'eau); L : extensibilité (en mm) correspondant au gonflement maximal de la pâte ; P/L : Rapport déterminant l'équilibre entre la ténacité et l'élasticité de la pâte ou rapport de configuration ; W : Travail de déformation de la pâte jusqu'à la rupture. Il caractérise la force de la farine ; ${ }^{1}$ et ${ }^{2}:$ (Cf. Tableau 1) ; - : Absence de données ; FR, F0, F1, F2, F3, F4 (Cf. Tableau 3). 


\section{DISCUSSION}

De l'analyse de ces résultats, on peut dire que les amidons étudiés, dans l'ensemble, sont très peu solubles dans l'eau à la température ambiante (à $25^{\circ} \mathrm{C}$ ). Evidemment, la cristallinité des amidons est due essentiellement aux macromolécules d'amylopectine dont la rupture est impossible sous l'action de l'eau à la température ambiante. Seuls, un traitement thermique plus ou moins rigoureux ou l'action d'un catalyseur peuvent rompre les liaisons des molécules d'amylopectine (Eerlingen et al., 1993). Généralement, la solubilité des amidons dépend de la modification de leur structure dont la sensibilité n'est révélée qu'à la suite d'un traitement thermique à $100{ }^{\circ} \mathrm{C}$, ou à l'aide d'un solvant (Buléon et al., 1990 ; Lefebvre et al., 2000). La faible solubilité à la température ambiante, en milieu aqueux, de l'amidon de la pomme de terre composé d'environ $25 \%$ d'amylose et $75 \%$ d'amylopectine pourrait en être la cause fondamentale (Godon, 1991; Boursier, 1993).

L'activité enzymatique des farines panifiables la plus importante, sur le plan technologique, est celle qui affecte l'amidon (Boursier, 1994 ; Cleary and Blakeney, 1992), c'est-à-dire l'activité amylolytique qui est caractérisée par la consistance d'un empois d'amidon. Elle est déterminée par le temps de chute de Hagberg (Mercier, 1985; Dobraszczyk and Morgenstern, 2003). En effet, il a été rapporté que plus le temps de chute est court, plus la consistance de l'empois est faible et plus grande est l'activité $\alpha$-amylasique (Godon et Willm, 1991 ; Dobraszczyk and Morgenstern, 2003). Deux types d'enzymes, les amylases $\alpha$ et $\beta$ participent à cette action. L'action de la $\beta$ amylase se traduit par une libération progressive de maltose, appréciée par la détermination du pouvoir diastasique de l'amidon. Elle dépend du taux d'endommagement de l'amidon (Lechon et Souppe, 1992 ; Boursier, 1994). De ce point de vue, il est fort probable que les écarts observés entre les temps de chute des amidons soient liés à la teneur en amylose et en amylopectine, à l'état d'endommagement des amidons, à la nature de l'enzyme, aux origines ou à l'état de pureté des amidons. De ce point de vue, pour les travaux ultérieurs, la détermination des teneurs en amylopectine et en amylose aussi bien dans les amidons étudiés que dans les farines reconstituées pourrait présenter un intérêt pour la présente étude.

On s'aperçoit que les valeurs des paramètres rhéologiques de tous les amidons analysés se présentent globalement avec des écarts relativement considérables, en comparaison avec les résultats rapportés par Boursier (1994). Ces écarts seraient également liés à la variabilité entre la granulométrie des amidons. Les travaux de Hounhouigan (1994) et Nago (1997) sur des maïs de différentes variétés ont révélé que les pâtes issues desdites farines sont d'autant plus visqueuses que les farines sont plus fines. La disparité observée pourrait être aussi liée au comportement de gélatinisation qui dépendrait de l'espèce botanique et du type cristallin de l'amidon déterminant le rapport entre les teneurs en amylopectine et en amylose (Boursier, 1994). La disparité est particulièrement remarquable au niveau des valeurs des viscosités des amidons au point de gélification, avec une forte prédominance de la viscosité de l'amidon de la pomme de terre, suivie de celle de l'amidon de maïs. L'amidon du blé a la plus faible viscosité de gélification. Les faibles valeurs de viscosité enregistrées au niveau des températures de gonflement pour tous les amidons seraient liées au fait qu'à cette température, l'éclatement des grains d'amidon n'a fait que commencer et n'aboutira à la formation de gel qu'à une température où l'éclatement des grains est maximal (Buléon et al., 1990). Concernant l'amidon de la pomme de terre, les phénomènes de gonflement par absorption d'eau et de solubilisation des macromolécules sont concomitants, alors que les amidons de céréales (polymorphe A) sont caractérisés par une première étape de gonflement limitée à la température de gélatinisation, suivie à $90{ }^{\circ} \mathrm{C}$ d'un second gonflement accompagné 
seulement d'une solubilisation plus marquée du contenu granulaire (Berton et al., 2002).

Ces variations observées entre les caractéristiques des amidons pourraient servir de critères pour une sélection variétale mais aussi de caractérisation des farines reconstituées, dans la mesure où les caractéristiques rhéologiques des amidons déterminent généralement celles des farines récurrentes (Lechon et Souppe, 1992 ; Uthayakumaran et al., 2002).

Il est apparu que la capacité d'hydratation des farines reconstituées croît avec l'augmentation des teneurs en eau et des quantités des prises d'essai. En outre, la farine F4 qui a la plus forte teneur en eau et dont la prise d'essai est la plus élevée, a la plus forte capacité d'hydratation. Toutefois, la teneur en eau de la farine de la pomme de terre F4 est supérieure à la valeur autorisée pour les farines panifiables (Roussel, 1991). Par ailleurs, Hoseney (1992) a rapporté que la capacité d'hydratation varie généralement entre 30 et $60 \%$. Par ailleurs, les pâtes des farines reconstituées ne sont pas stables, le temps de stabilité à la limite de 500 UB étant inférieur à une minute, contrairement à celui de la farine de référence qui est en moyenne de 8 minutes.

En outre, on s'est rendu compte que plus le temps de stabilité augmente, plus la courbe de farinographe tend vers la limite inférieure de 500 UB et plus les pâtes deviennent molles et très collantes. Le temps de stabilité des pâtes des farines est également fonction des méthodes de caractérisation utilisées (Lechon et Souppe, 1992 ; Rouillé et al., 2005). En effet, deux méthodes ont été utilisées pour la détermination des caractéristiques rhéologiques des pâtes des farines reconstituées. Il s'est révélé que les pâtes obtenues à partir des farines reconstituées au cours du pétrissage n'ont pas pu être extraites lorsque nous avons utilisé la méthode qui repose sur la mesure de la quantité d'eau nécessaire au pétrissage, en fonction des teneurs en eau des farines. En réalité, l'évacuation à travers l'orifice de l'extracteur du pétrin est impossible, du fait de la dureté des pâtes. Par conséquent, les essais au relaxo-calculateur conditionnés, par l'extraction des pâtons, en vue de la détermination de la force, de l'extensibilité, du gonflement et de la ténacité des pâtes étaient impossibles. De même, lorsque nous avons appliqué la méthode qui consistait à utiliser, pour chaque farine reconstituée, la quantité d'eau déterminée au farinographe Brabender, sur la base de la capacité d'hydratation des pâtes, nous avons obtenu des pâtes trop liquides et collantes qui rendent impossibles la détermination des caractéristiques rhéologiques.

Ainsi, seules les caractéristiques rhéologiques de la pâte de la farine F1 ont pu être mesurées. La valeur de la force de la farine $\left(\mathrm{W}=85,10.10^{-4}\right)$ caractérisant le travail de la déformation de la pâte jusqu'à la rupture est proche des valeurs recommandées pour les pâtes des farines panifiables qui sont comprises entre 90 et $10010^{-4} \mathrm{~J}$ (Godon et Willm, 1991 ; Rouillé et al., 2005). Le rapport de configuration $(\mathrm{P} / \mathrm{L}=0,88)$ traduisant l'équilibre entre la ténacité et l'élasticité de la pâte s'écarte sensiblement de la norme qui est comprise entre 0,3 et 0,4 (Godon et Willm, 1991 ; Berton et al., 2002 ; Uthayakumaran et al., 2002). Les écarts observés par rapport à la farine Fo seraient dus aux amidons dont les origines sont supposées avoir une influence sur les caractéristiques rhéologiques des farines. En outre, les résultats des travaux de Petrofsky et Hoseney (1995) et de Uthayakumaran et al. (2002) ont révélé que des amidons isolés de différentes variétés de blé et mélangés avec différentes variétés de gluten présentent des caractéristiques rhéologiques différentes. Une correction des farines par addition d'amylases ou d'agents améliorants ou l'utilisation d'un résistographe pour un pétrissage intensifié ou d'un alvéographe à consistance constante, sur différentes farines composées à base de différentes variétés et de diverses sources d'amidon et de gluten ont amélioré les résultats.

En effet, au terme de la correction, les pâtes obtenues avec de la farine reconstituée 
$\mathrm{F} 1$ et $\mathrm{F} 3$ présentent, au cours du pétrissage, un aspect plus souple et plus consistant se rapprochant, plus ou moins, des caractéristiques de la pâte issue de la farine Fo. La pâte de la farine F4 est plus dure, au cours du pétrissage. De façon générale, la qualité des pâtes s'améliore, avec le temps de pointage (temps de repos), notamment, pour les pâtes des farines Fo, F1 et F3. A la fin du pointage, une augmentation sensible du volume des pâtes a été enregistrée. Cette amélioration serait liée aux effets des ingrédients utilisés, notamment la margarine composée d'agents assouplissants des pâtes, tels que la lécithine, les mono et les diglycérides. Le développement des pâtes serait la conséquence d'une double fermentation provoquée par la levure chimique utilisée, subséquente au sucre incorporé (Manohar et Rao, 1997) et les enzymes des farines. Le gluten aurait retenu une partie du $\mathrm{CO}_{2}$ produit au cours de cette formation qui pourrait compenser l'affaiblissement des pâtes des farines reconstituées. Néanmoins, alors que les pâtes des autres farines sont plus collantes et deviennent plus dures à la fin de la mise en forme, les pâtes des farines Fo, F1 et F3 sont plus souples et plus faciles à manipuler.

\section{Conclusion}

La présente étude a fourni des résultats intéressants sur les propriétés physicochimiques, rhéologiques des farines reconstituées à partir des propriétés des amidons récurrents. Cependant, la détermination des teneurs en amylopectine et en amylose des amidons étudiés permettra de mieux comprendre leurs caractéristiques rhéologiques et les interactions amidongluten. Par ailleurs, en utilisant l'alvéographe de Chopin et le farinographe de Brabender, il était impossible de prédire les propriétés panifiantes des farines reconstituées à partir des propriétés des amidons qui ont servi à leur formulation, étant donné que les résultats obtenus n'ont pu établir une corrélation systématique de cause à effet entre les propriétés des amidons utilisés et celles des farines reconstituées. Malgré que la farine de blé classique soit constituée de $90 \%$ d'amidon et de $10 \%$ de gluten, il est apparu clairement qu'on ne saurait ajouter du gluten vital de blé à n'importe quel amidon même dans les proportions précédemment indiquées pour obtenir des farines panifiables sans correction avec les caractéristiques rhéologiques requises. En revanche, les caractéristiques rhéologiques des pâtes issues des farines reconstituées pourraient être nettement améliorées si on utilisait un rhéomètre pour un pétrissage intensifié, un résistographe ou un alvéographe à consistance constante.

\section{REMERCIEMENTS}

Les auteurs expriment leur gratitude à la Communauté Universitaire Belge pour le Développement (CUD) pour l'appui financier. Nous remercions le Laboratoire de l'Unité de Technologie des Industries Alimentaires de la Faculté Universitaire des Sciences Agronomiques de Gembloux en Belgique de l'appui technique.

\section{REFERENCES}

AACC (American Association of Cereal Chemist). 1983. Approved Methods of the AACC: Method 30-25, approved 1983; Method 54-30; Method 44-15 approved October 1983. The Association: St Paul, $\mathrm{MN}$.

AACC (American Association of Cereal Chemist). 1984. Approved Methods of the AACC: Method 44-15 A, approved October 1975, revised October 1981, Method 54-21, approved October 1984. The Association : St Paul, MN.

AFNOR (Association Française de Normalisation). 1991. NF V03-703; NF V03-707; NF V03-720. Recueil des Normes Françaises. AFNOR, Tour Europe 92049, Paris la Défense Cedex.

Asiedu JJ. 1991. La Transformation des Produits Agricoles en Zone Tropicale. Approche Technologique. Editions KARTHALA et CTA ; 332.

Balla A, Blecker C, Oumarou M, Paquot M, Deroanne C. 1999. Mise au point de pains 
composites à base de mélanges de farines de sorgho-blé et analyse texturale. Biotechnol. Agron. Soc. Environ., 3(2): 69-77.

Berton B, Scher J, Villieras F, Hardy J. 2002. Measurement of hydration capacity of wheat flour. Influence of composition and physical characteristics. Powder Tech., 128(2-3): 326-331.

Boursier B. 1993. Les Amidons Modifiés Chimiquement dans la Filière Alimentaire. Epaississants et Gélifiants. ADRIA : Vannes ; 10 -11.

Boursier B. 1994. Applications alimentaires des amidons modifiés. Actualités Techniques et Industrielles. Ind. Alim. Agric, 10: 583-591.

Buléon A, Colonna P, Leloup V. 1990. Les amidons et leurs dérivés dans les industries de céréales. Ind. Alim. Agric., 6: 515-532.

Chaunier L, Chiron H, Della Valle G, Réguerre A-L, Desse T. 2005. Mesure des propriétés élongationnelles de pâtes à pain et évaluation de la texture des mies par analyse d'images. Rhéologie, 8: 13-19.

Cleary BV, Blakeney AB. 1992. Mesure de l'activité alpha-amylasique des farines des céréales. Neuvième Congrès International des Céréales et du Pain. Résumé des sessions techniques. Industries des céréales - Revue de l'APIC, 1-5 juin, Paris, France, p 50.

CTA (Centre Technique de Coopération Agricole et rurale). 1991. Valorisation technologique des céréales tropicales par des procédés de fabrication industrielle des pâtes alimentaires et de produits locaux. Science et Technique au Service du développement. Deuxième Programme, 1, 223-228.

Dobraszczyk BJ, Morgenstern, MP. 2003. Review: rheology and the breadmaking process. J. Cereal Sci., 38: 229-245.

Defloor I. 1995. Factors governing the breadmaking potential of cassava (Manihot esculenta crantz). $\mathrm{PhD}$ thesis. University of K.W.L., Leuven, Belgium, p 155.
Eerlingen RC, Deceuninck M, Delcour JA. 1993. Enzyme- Resistant starch: The influence of amylose chain length on resistant starch formation. Cereal. Chem., 70: 345-350.

Godon B. 1991. Biotransformation des Produits Céréaliers. Collection Science et Technique Agro-alimentaire. Lavoisier: Paris, France ; 221.

Godon B, Willm C. 1991. Les Industries de Première Transformation des Céréales. Collection Technique et Documentation. Lavoisier : Paris, France ; 679.

Hoseney RC. 1992. Interaction entre les protéines et l'amidon dans la pâte et dans le pain. Neuvième Congrès International des Céréales et du Pain. Résumé des sessions techniques. 1-15 juin, Paris. Industries des céréales- Revue de 1'APIC, p 50.

Hounhouigan DJ. 1994. Fermentation of maize (Zea mays L.) meal for mawè production in Bénin: Physical, chemical and microbiological aspects, $\mathrm{PhD}$ thesis. Agricultural University, Wageningen, The Netherlands, p 83.

Lefebvre J, Popineau Y, Deshayes G, Lavenant L. 2000. Temperature-induced changes in dynamic rheological behaviour and size distribution of polymeric proteins for glutens from wheat near-isogenic lines differing in HMW glutenin subunit composition. Cereal Chem., 77: 193-201.

Lechon V, Souppe J. 1992. L'amidon endommagé. Les enzymes sont concernées. Neuvième Congrès International des Céréales et du Pain. Résumé de la session des posters. Industries des céréales- Revue de 1'APIC, 1-5 juin, Paris, France, p 43.

Manohar RS, Rao PH. 1997. effects of sugars on the rheological characteristics of biscuits dough and quality of biscuits. $J$. Sci. Food Agric., 75: 383-390.

Melcion JP. 1991. Le mélange des matières pulvérulentes. In Les Industries de Premières Transformations des Céréales, Collection Technique et Documentation, 
Godon B, Willm C. (eds). Lavoisier : Paris, France ; 312-329.

Mercier C. 1985. Les enzymes amylolitiques. In Hydrolases et Dépolymérases, Mouranche A, Costes C (eds). GauthierVillars : Paris ; 109-142.

Mestres C. 1986. Gélification d'amidon de maïs modifié thermiquement. Application à la fabrication des pâtes alimentaires sans gluten. Thèse, Montpellier, France.

Nago CM. 1997. La transformation alimentaire traditionnelle du maïs au Bénin : détermination des caractéristiques physico-chimiques des variétés en usage; relations entre l'obtention et la qualité des principaux produits dérivés. Thèse de Doctorat d'Etat, Paris, France, p 201.

Petrovsky KE, Hoseney RC. 1995. Rheological properties of dough made with starch and gluten from several cereal sources. Cereal Chem., 72: 53-58.

Rouillé J, Della Valle G, Lefebvre J, Sliwinski E, van Vliet T. 2005. Shear and extensional properties of bread doughs affected by their minor components. $J$. Cereal Sci., 42: 45-57.

Roussel P. 1991. Amélioration de la qualité des farines: Additifs autorisés pour la fabrication $\mathrm{du}$ pain français. In Les Industries de Première Transformation des Céréales, Collection Technique et Documentation, Godon B, Willm C (eds). Lavoisier : Paris, France ; 591-625.

Uthayakumaran S, Newberry M, Phan Thien N, Tanner R. 2002. Small and large strain rheology of wheat gluten. Rheol. Acta, 41: 162-172. 